\title{
ANÁLISE DO PADRÃO HIDRODINÂMICO DE UM BIORREATOR PARA PRODUÇÃO DE HIDROGÊNIO
}

\author{
A. A. LONDERO ${ }^{1}$, L. M. PORTO ${ }^{1}$ \\ ${ }^{1}$ Universidade Federal de Santa Catarina, Departamento de Engenharia Química e Engenharia de \\ Alimentos \\ E-mail para contato: \{afonso; luismar\}@intelab.ufsc.br
}

\begin{abstract}
RESUMO - A produção biológica hidrogênio é considerada como uma alternativa atrativa para geração de energia em oposição ao uso de combustíveis fósseis. Entretanto, para alcançar viabilidade econômica é necessário um expressivo progresso tecnológico. Estudos presentes para melhoria da eficiência estão focados principalmente em características biológicas e químicas e em uma menor extensão na influência da hidrodinâmica de biorreatores. $\mathrm{O}$ presente estudo investiga a dinâmica dos fluxos em um biorreator utilizando ferramentas de Fluidodinâmica Computacional (CFD). Um biorreator de 5 L foi modelado como um reator de tanque agitado equipado com dois impelidores. A distância entre os impelidores e a velocidade de agitação foram variadas e observou-se mistura mais eficiente para a configuração com a maior distância entre os impelidores.
\end{abstract}

\section{INTRODUÇÃO}

A produção biológica hidrogênio como alternativa energética aos combustíveis fósseis tem sido amplamente considerada devido ao seu alto conteúdo energético e baixa emissão de poluentes. Entre as diferentes formas de se conduzir a produção de biohidrogênio, destaca-se a fermentação anaeróbica pelo seu maior rendimento e possível utilização de resíduos industriais ou agrícolas como substrato (Das e Veziroglu, 2008). Embora a produção biológica de hidrogênio tenha sido demonstrada com sucesso em escala laboratorial, a tecnologia ainda deve ser explorada para competir com a produção industrial a partir de resíduos fósseis, considerando-se a viabilidade econômica, eficiência e confiabilidade.

O processo de produção de biohidrogênio envolve complexos mecanismos químicos, biológicos e físicos com múltiplas interações entre fases fluidas e sólidas. Fatores como inóculo, substrato, pH, nutrientes e temperatura estão entre os parâmetros mais estudados para otimização de processos fermentativos para produção de hidrogênio (Wang e Wan, 2009). O estudo das características físicas do escoamento em biorreatores, entretanto, tem sido cada vez mais explorado devido à possibilidade de otimizar a produção de biohidrogênio através da interação entre os fenômenos químicos, biológicos e físicos no domínio reacional. Além disso, o bom entendimento das características hidrodinâmicas é um precursor obrigatório para o aumento de escala de qualquer processo biotecnológico.

A fermentação anaeróbica para produção de hidrogênio é comumente estudada em tanques 
agitados em diversos modos de operação, como contínuo (CSTR), semi-contínuo, batelada, entre outros. Do ponto de vista de engenharia, o modo contínuo apresenta produtividade mais alta, mas sofre de problemas como diluição de biomassa ou substrato, que são fortemente influenciados pelo comportamento hidrodinâmico do biorreator (Show et al., 2011). A operação em batelada reduz o risco de contaminação e de diluição mas em geral apresenta taxas de produção inferiores. Conforme o tamanho do biorreator aumenta, problemas como zonas mortas, agitação ineficiente e heterogeneidade da mistura podem comprometer significativamente a produção de hidrogênio. $O$ emprego de velocidades muito altas de agitação, a fim de melhorar a homogeneidade do sistema, pode causar inatividade ou morte celular por cisalhamento e consequente fracasso da operação (Wang et al., 2010).

Softwares modernos de Fluidodinâmica Computacional (CFD) são capazes de simular o comportamento hidrodinâmico, transferência de massa e calor, reações químicas, interações multifásicas e outros fenômenos ocorrendo em biorreatores através da solução das diversas equações matemáticas que descrevem esses fenômenos. Um modelo computacional bem estabelecido de um processo biotecnológico permite que se avalie e otimize a configuração de biorreatores sem a necessidade de investir massivamente em testes experimentais (Ding et al., 2010; Wang et al., 2014).

O presente trabalho tem como objetivo realizar um estudo preliminar do comportamento fluidodinâmico de um biorreator com uma modelagem multifásica, a fim de estabelecer um modelo computacional de um biorreator de escala laboratorial para produção de hidrogênio ou de outros metabólitos de interesse. Os perfis de velocidade e fração volumétrica foram avaliados para três velocidades de agitação (150, 180 e $210 \mathrm{rpm})$ e três configurações de biorreator, onde a distância entre os impelidores foi modificada.

\section{METODOLOGIA}

\subsection{Configuração do biorreator}

O tanque agitado utilizado nas simulações foi modelado com base no biorreator BioFlo 110 (New Brunswick, USA), com 5 L de volume útil e agitado por duas turbinas do tipo Rushton. As dimensões do biorreator estão demonstradas na Figura 1. Simulações foram realizadas com a distância entre os impelidores iguais a $7,7 \mathrm{~cm}$ (configuração padrão do biorreator, neste trabalho denominada configuração A), 10,7 cm (configuração B) e 13,7 cm (configuração C), cada um avaliado com velocidades de agitação de 150, 180 e 210 rpm. 


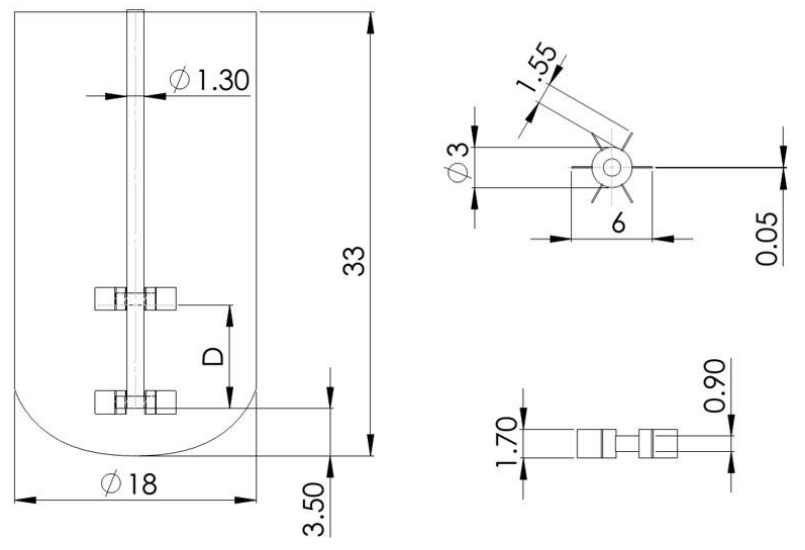

Figura 1 - Dimensões (em cm) do biorreator utilizado nas simulações.

\subsection{Metodologia computacional}

Modelo computacional: Os fenômenos de transferência de quantidade de movimento, energia e massa são descritos por equações matemáticas que são discretizadas e resolvidas iterativamente para cada volume de controle. Dessa maneira, uma aproximação dos valores de cada variável é definida em pontos específicos do domínio e inter-relacionada com os volumes de controle vizinhos através das fronteiras do volume. O modelo de turbulência utilizado foi o modelo padrão de k- $\epsilon$, que é o modelo mais utilizado na modelagem de escoamentos turbulentos devido à sua simplicidade, baixo requerimento computacional e facilidade de convergência (Tamburini et al., 2013). A fim de simplificar a análise hidrodinâmica do biorreator, a fase líquida consiste em água pura e a fase gasosa, ar. O reator opera em modo batelada, sem entrada e saída de água e ar e em estado estacionário. Para simular o comportamento multifásico foi o utilizado o modelo Euleriano - Euleriano Homogêneo, onde todas as quantidades de transporte (exceto a fração volumétrica) do processo são mantidas iguais para cada fase. Dessa forma, a equações de transporte são resolvidas em uma forma geral ao invés de individualmente para cada fase. As equações da conservação da quantidade de movimento (Eq. 1) e massa (Eq. 2) são definidas como (Ansys, 2012):

$$
\begin{aligned}
& \frac{\partial(\rho \mathrm{U})}{\partial t}+\nabla \cdot\left(\rho \mathrm{U} \times \mathrm{U}-\mu\left(\nabla \mathrm{U}+(\nabla \mathrm{U})^{\mathrm{T}}\right)\right)=S_{M}-\nabla p \\
& \frac{\partial\left(r_{\alpha} \rho_{\alpha}\right)}{\partial t}+\nabla \cdot\left(r_{\alpha} \rho_{\alpha} \mathrm{U}_{\alpha}\right)=S_{M S \alpha}+\sum_{\beta=1}^{N_{p}} \Gamma_{\alpha \beta}
\end{aligned}
$$

Onde

$$
\begin{aligned}
& \rho=\sum_{\alpha=1}^{N_{p}} r_{\alpha} \rho_{\alpha} \\
& \mu=\sum_{\alpha=1}^{N_{p}} r_{\alpha} \mu_{\alpha}
\end{aligned}
$$




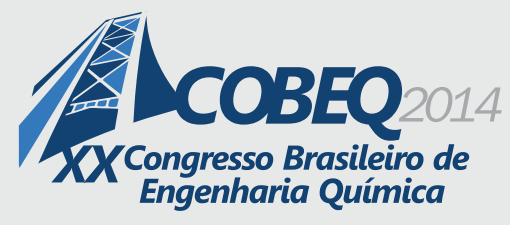

19 a 22 de outubro de 2014
Florianópolis/SC

Domínio computacional e condições de contorno: O método MRF (Multiple Reference Frame), comumente utilizado para modelar reatores agitados, foi utilizado para simular o movimento dos dois impelidores. A abordagem do método MRF consiste em modelar os impelidores em domínios de referência rotativos e a região externa (restante do tanque) em um domínio estacionário (Taghavi et al., 2011). Para integrar os domínios estacionário e rotativos, foi adotada a interface Frozen Rotor, que conserva o transporte das quantidades físicas através das fronteiras enquanto mantém a posição relativa de ambos os domínios. O remanescente do tanque foi dividido em dois domínios com a finalidade de inicializar as concentrações de ar e água: um domínio com a fração volumétrica inicial de ar igual a 1, em contato com a atmosfera, de $7 \mathrm{~cm}$ de altura; e o restante do tanque, com fração inicial de água igual a 1. As forças de empuxo foram implementadas pela força gravitacional alinhada ao eixo do rotor e pela diferença de densidade entre as fases, com a densidade de referência igual ao do fluido menos denso (ar).

As condições de contorno em todas as superfícies sólidas (impelidor, eixo, paredes) foram definidas como condições de parede sem escorregamento na superfície. À superfície de contato entre o ar e a água foi definida uma interface com fluxo conservativo entre os domínios, enquanto na superfície livre do tanque uma condição de abertura com pressão constante de 1 atmosfera foi definida. A temperatura foi assumida como uniforme em todo o domínio.

Solução numérica: A geometria do biorreator foi modelada utilizando o software SolidWorks 2013 e exportada para o software ANSYS ICEM CFD, onde foi gerada a malha computacional. O domínio do tanque constitui-se de 500452 elementos e dos impelidores, de 871489 elementos cada. A densidade de elementos foi maximizada nas regiões de maior contraste de velocidades, como nas pás dos impelidores, na interface água - ar e nas paredes do tanque.

O software comercial de solução de problemas de fluidodinâmica computacional ANSYS CFX 13.0 foi utilizado para resolver as equações governantes do modelo. A simulações foram realizadas em um computador com processador Intel Core i3 $3.30 \mathrm{GHz}$ com $8.00 \mathrm{~Gb}$ de memória RAM. Os esquemas advectivo e de turbulência foram selecionados como de alta resolução e de primeira ordem, respectivamente, com um controle automático do passo do tempo. O critério de convergência com máximo de $10^{-4}$ para os resíduos das soluções numéricas das equações (Root Mean Square) foi utilizado. Para garantir a conservação de massa no domínio, os volumes de ar e água totais no biorreator foram computados a cada iteração e observou-se um desvio menor que $2 \%$ para todas as simulações.

\section{RESULTADOS E DISCUSSÃO}

O campo de velocidades na seção transversal ao longo do eixo está plotado na forma de vetores na Figura 2 para as três configurações e velocidades de agitação de $210 \mathrm{rpm}$. Um padrão geral de fluxo pode ser observado para as todas as situações, no qual um fluxo intenso é gerado pelas pás das turbinas impulsionando o fluido paralelamente ao eixo das pás. O fluxo se divide em dois e se direciona para cima ou para baixo ao longo da parede, formando regiões de recirculação devido à baixa pressão na região imediatamente superior e inferior aos impelidores. Os perfis de fluxo se assemelham a padrões encontrados em biorreatores similares e em tanques maiores agitados por impelidores do tipo Rushton (Um e Hanley, 2008; Taghavi et al., 2011; Kulkarni e Patwardhan, 2013). 


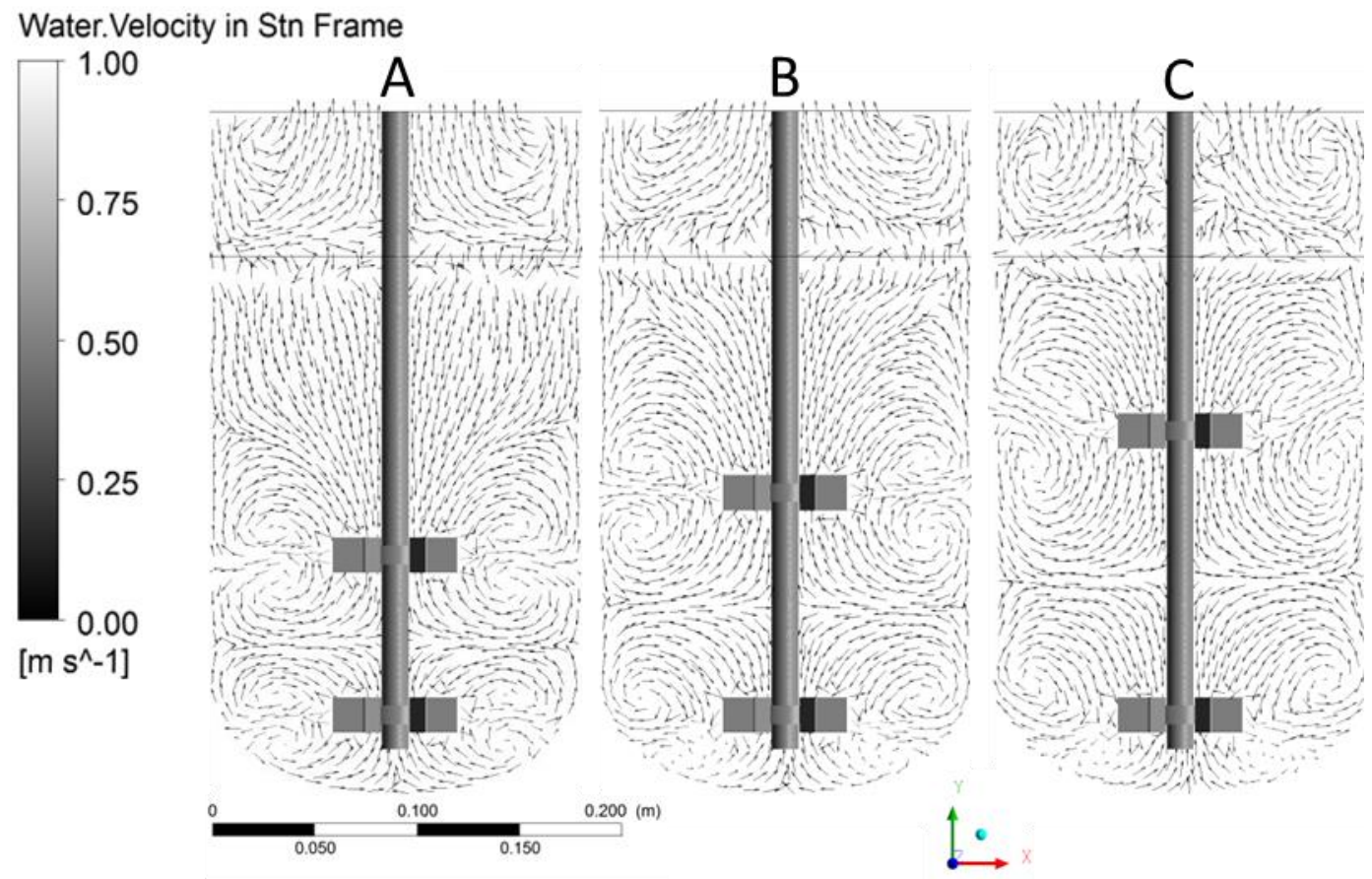

Figura 2 - Campos de vetores de velocidade para biorreatores agitados a 210 rpm, com distância entre as turbinas igual a 7,7 cm (A), 10,7 cm (B) e 13,7 cm (C), avaliados no plano de seção transversal vertical de simetria.

A Figura 3 apresenta contornos da velocidade da água para cada configuração e velocidade de agitação. A velocidade máxima de água encontrada foi de $0.55,0.67$ e $0.80 \mathrm{~m} / \mathrm{s}$ para as velocidades de agitação de 150, 180 e $210 \mathrm{rpm}$, respectivamente. O campo de velocidades indica que as turbinas operam essencialmente de forma independente e cada uma gera seus próprios vórtices de recirculação superiores e inferiores. Esse comportamento é reportado para sistemas monofásicos ou multifásicos com duas ou mais turbinas do tipo Rushton (Kerdouss et al., 2006; Taghavi et al., 2011) Observa-se que o aumento da velocidade de agitação promove uma melhoria na homogeneidade do meio reacional, principalmente na direção radial, mas não interfere significativamente na mistura ao longo do eixo. Em contraste, o aumento da distância entre as turbinas promove uma distribuição axial de velocidades mais eficiente mesmo para rotações mais baixas, como pode ser visto para as configurações $\mathrm{B}$ e C a $150 \mathrm{rpm}$. Nas configurações B e C, o líquido se mistura com mais eficiência no fundo do tanque, característica desejável na operação de biorreatores onde o depósito de biomassa ou formação de flocos é um fator importante. 


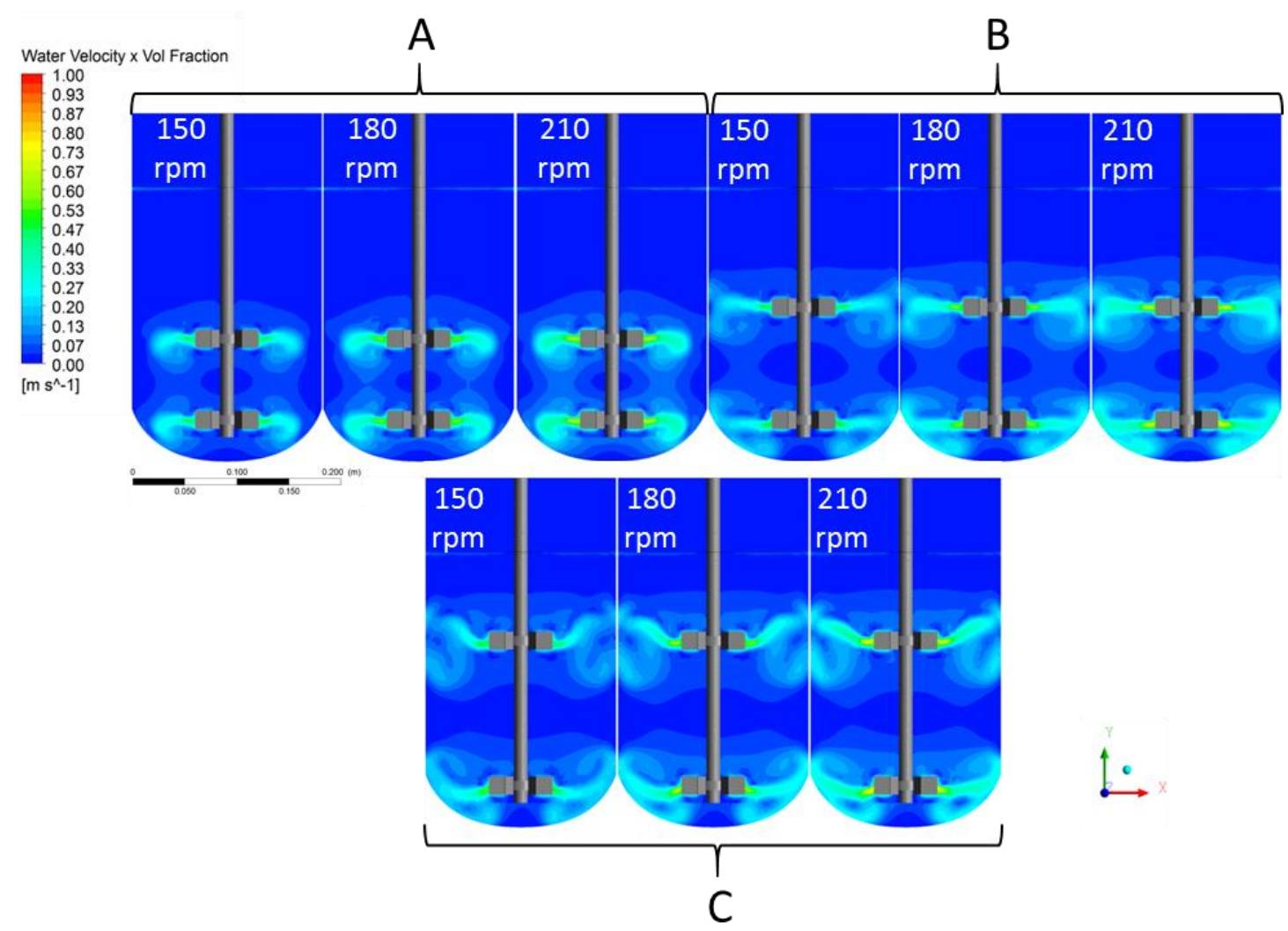

Figura 3 - Contornos de velocidade para biorreatores agitados a 150, 180 e $210 \mathrm{rpm}$, com distância entre as turbinas igual a 7,7 cm (A), 10,7 cm (B) e 13,7 cm (C), avaliados no plano de seção transversal vertical de simetria.

A Figura 4 mostra o detalhe da fração volumétrica na interface entre o domínio líquido e gasoso para a configuração $\mathrm{C}$ com velocidade de agitação de $210 \mathrm{rpm}$. Para todas as situações o perfil de concentração de água e ar é parecido, com difusão entre as fases mas sem a formação de vórtice que é característica desse tipo de operação. Modelos mais sofisticados que aproximam as interações entre fases com maior precisão como Large Eddy Simulation (LES), Volume of Fluid (VOF) podem ser aplicados ao modelo atual para capturar a formação de vórtices na superfície do líquido (Torré et al., 2007; Kulkarni e Patwardhan, 2013). O estudo da formação de vórtices pode ser especialmente interessante no processo de produção biológica de hidrogênio por proporcionar um aumento na área de contato entre líquido e gás e promover uma dissociação mais eficiente de hidrogênio.

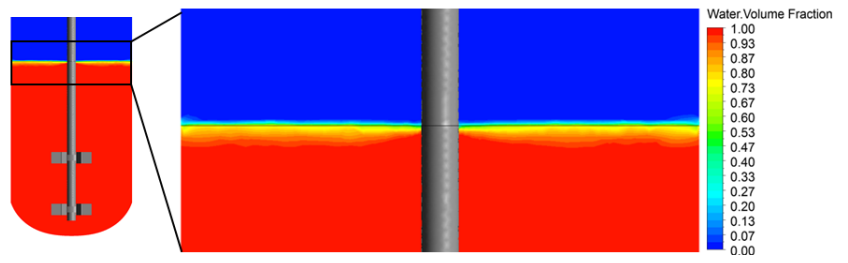

Figura 4 - Detalhe da interface ar-água para a configuração C, a 210 rpm 


\section{CONCLUSÕES}

O perfil hidrodinâmico de um reator tipicamente utilizado para fermentação em escala laboratorial foi avaliado para diferentes configurações de distância entre impelidores e velocidade de agitação. As configurações de biorreator com impelidores distantes entre si por 13,7 e 10,7 cm demonstraram mistura axial mais eficiente se comparados à configuração padrão de 7,7 cm de distância e promoveram agitação mais eficiente no fundo do reator. $\mathrm{O}$ modelo homogêneo Euleriano demonstrou robustez e rápida convergência, mas para o caso de simulação de operações com formação de vórtice se torna necessária a utilização de modelos mais sofisticados para simular as interações multifásicas. A utilização de simetria geométrica no modelo a fim de reduzir o tempo computacional também deve ser avaliada, bem como a otimização do número de elementos da malha computacional. Um modelo hidrodinâmico computacional bem consolidado pode então ser validado experimentalmente e acoplado a um modelo cinético para simular o processo de produção de hidrogênio e crescimento celular.

\section{NOMENCLATURA}

$\begin{array}{ll}M & \text { Número de Mach local } \\ N_{p} & \text { Número de fases presentes no sistema } \\ p & \text { Pressão estática (termodinâmica) }\left(\mathrm{kg} \mathrm{m}^{-1} \mathrm{~s}^{-2}\right) \\ r & \text { Fração volumétrica } \\ S_{M} & \text { Fonte de quantidade de movimento }\left(\mathrm{kg} \mathrm{m}^{-2} \mathrm{~s}^{-2}\right) \\ S_{M S} & \text { Fonte de massa }\left(\mathrm{kg} \mathrm{m}^{-3} \mathrm{~s}^{-1}\right) \\ t & \text { Tempo (s) } \\ \mathrm{U} & \text { Vetor de velocidades }\left(\mathrm{m} \mathrm{s}^{-1}\right) \\ \alpha & \text { Subscrito referente à fase } \alpha \\ \beta & \text { Subscrito referente à fase } \beta \\ \Gamma & \left.\text { Difusividade (kg m } \mathrm{s}^{-1}\right) \\ \mu & \text { Viscosidade dinâmica }\left(\mathrm{kg} \mathrm{m}^{-1} \mathrm{~s}^{-1}\right) \\ \rho & \text { Densidade }\left(\mathrm{kg} \mathrm{m}^{-3}\right)\end{array}$

\section{REFERÊNCIAS}

ANSYS, Inc. ANSYS CFX 14.5 - Pre User's Guide. 2012. 
DAS, D.; VEZIROGLU, T. N. Advances in biological hydrogen production processes. Int. J. Hydrogen Energy, v. 33, n. 21, p. 6046-6057, 2008.

DING, J.; WANG, X.; ZHOU, X-F.; REN, N-Q.; GUO, W-Q. CFD optimization of continuous stirred-tank (CSTR) reactor for biohydrogen production. Bioresource Technol., v. 101, n. 18, p. 7005-7013, 2010.

KERDOUSS, F.; BANNARI, A.; PROULX, P. CFD modeling of gas dispersion and bubble size in a double turbine stirred tank. Chem. Eng. Science, v. 61, n. 10, p. 3313-3322, 2006.

KULKARNI, A. L.; PATWARDHAN, A. W. CFD modeling of gas entrainment in stirred tank systems. Chem. Eng. Res. Des., 2013.

SHOW, K.-Y.; LEE, D.-J.; CHANG, J.-S. Bioreactor and process design for biohydrogen production. Bioresource Technol., v. 102, n. 18, p. 8524-8533, 2011.

TAGHAVI, M.; ZADGHAFFARI, R.; MOGHADDAS, J.; MOGHADDAS, J. Experimental and CFD investigation of power consumption in a dual Rushton turbine stirred tank. Chem. Eng. Res. Des., v. 89, n. 3, p. 280-290, 2011.

TAMBURINI, A.; CIPOLLINA, A.; MICALEA,G.; BRUCATO, A.; CIOFALO, M. Influence of drag and turbulence modelling on CFD predictions of solid liquid suspensions in stirred vessels. Chem. Eng. Res. Des., n. 0, 2013.

TORRÉ, J.-P.; FLETCHER, D.; LASUYE, T.; XUEREB, C. Single and multiphase CFD approaches for modelling partially baffled stirred vessels: Comparison of experimental data with numerical predictions. Chem. Eng. Science, v. 62, n. 22, p. 6246-6262, 2007.

UM, B.-H.; HANLEY, T. A CFD model for predicting the flow patterns of viscous fluids in a bioreactor under various operating conditions. Korean J. Chem. Eng., v. 25, n. 5, p. 10941102, 2008/09/01 2008.

WANG, H. et al. CFD modeling of hydrodynamic characteristics of a gas-liquid two-phase stirred tank. Appl. Math. Model., v. 38, n. 1, p. 63-92, 2014.

WANG, J.; WAN, W. Factors influencing fermentative hydrogen production: A review. Int. J. Hydrogen Energy, v. 34, n. 2, p. 799-811, 2009.

WANG, X. et al. Scale-up and optimization of biohydrogen production reactor from laboratoryscale to industrial-scale on the basis of computational fluid dynamics simulation. Int. J. Hydrogen Energy, v. 35, n. 20, p. 10960-10966, 2010. 